\section{Testing the forecast of aftershocks: a simple method with an example of application}

\author{
Changsheng Jiang, Zhongliang Wu \\ Institute of Geophysics, China \\ Earthquake Administration, Beijing, \\ People's Republic of China
}

\begin{abstract}
Tests of aftershock forecast have to consider the temporal distribution of aftershocks, which differ from mainshocks. This paper uses as an example the 2008 Wenchuan aftershock sequence and the load-unload response ratio forecast of aftershocks. We discuss a simplified test scheme using the Ogata-transformed time to make the transformed aftershock sequence homogenously random. While an example was taken using the Molchan error diagram, other testing schemes are also valid for the transformed aftershock sequence.
\end{abstract}

\section{Introduction}

Forecast of the probability, or the time of increased probability (TIP) of strong aftershocks, deals with a limited spatial range and specific temporal duration. It is a special form of earthquake forecast with theoretical feasibility and practical implications. In statistical seismology, one of the critical issues is how to test the statistical significance of an earthquake forecast scheme against real seismic activity. ${ }^{1-5}$ What is important is that the test method has to consider the water-level seismicity, otherwise results will be misleading. ${ }^{6}$

Aftershock sequence is intrinsically different from mainshock series, as described by the Omori-Utsu-law-type attenuation ${ }^{7}$ and this has to be taken into consideration when testing aftershock forecast. In this paper, we discuss a simplified approach to this problem based on the existing tools of aftershock analysis and earthquake forecast. These were applied to an analysis of the Wenchuan aftershock sequence and the forecast of aftershocks based on the load-unload response ratio (LURR) approach. ${ }^{8}$

\section{Characterizing an aftershock sequence for the test of aftershock forecast}

The basic idea for the test of a forecast scheme is to rule out the null hypothesis of possible random correlation between the forecast and the occurrence of earthquakes. For this purpose, several methods have been wide- ly used in testing the forecasts either in the form of probability ${ }^{9}$ or in the form of black-andwhite alarms. ${ }^{10}$ For a mainshock series, a working assumption that is often used is that earthquakes are randomly distributed in time. It is well known that the aftershock sequence is not homogeneously distributed along the natural time axis. It is also well known that the more frequent the earthquakes, the higher the probability for random hit, a factor that has to be taken into consideration when testing aftershock forecast.

Aftershocks generally attenuate according to Omori-Utsu's law. ${ }^{7}$ Given this, in testing aftershock forecast, the alarm time can be measured not along the natural time axis but along the transformed time axis, so that along the transformed time axis the aftershocks are homogeneously random. The Ogata-transformed time ${ }^{11}$ provides a convenient tool for such a transformation. The main feature of the Ogata-transformed time and its application to the forecast of aftershocks can be summarized as follow: i) the transformed time converges to natural time $t$ when $t$ becomes very long; ii) different aftershock sequences have different transformations, depending on the properties of the aftershock sequences; iii) aftershock sequence becomes homogeneously random along the transformed time axis; and iv) measuring the alarms along the transformed time axis, previous testing schemes for mainshock series can be directly applied to an aftershock sequence.

This idea of deforming the time axis to have a randomly distributed earthquake sequence is not new. In estimating the magnitude of completeness of an earthquake catalogue, orders of occurrence, rather than the origin time of earthquakes, are used to avoid the effect of the temporal heterogeneity of the earthquake sequence. ${ }^{12}$ In the studies identifying anomalous quiescence/activation from the mainshock and aftershock series, the same idea was applied in an analysis of the earthquake catalogue. ${ }^{11,13,14}$ In particular, in the study by Matsu'ura, ${ }^{13}$ a frequency-linearized time scale was used based on the modified 0mori formula, which is almost identical to the transformed-time scale discussed here. The SASeis2006 software package (http//bemlar. ism.ac.jp/www2/SASeisUpCollection/SASeis20 06/) can be directly used for obtaining the transformation.

It is worth pointing out that in the testing methods, such as the ROC test ${ }^{9}$ and/or the Molchan error diagrams,,$^{15}$ as well as in other tests, in principle it is not necessary to assume that earthquakes are randomly distributed to compare the forecast with a random hit, i.e. considering the inhomogeneous distribution of earthquakes, the statistical test can still be conducted using a deformed parameter space. Our simplified approach does not, therefore,
Correspondence: Zhongliang Wu, Institute of Geophysics, China Earthquake Administration, 100081 Beijing, People's Republic of China.

E-mail: wuzl@cea-igp.ac.cn

Key words: aftershock, forecast, ETAS, LURR, Wenchuan earthquake.

Acknowledgments: our thanks go to Y. Ogata, J.C. Zhuang, and J. Zechar for their help in understanding and using the statistical tools for aftershock analysis. LURR data and information about the LURR forecast are from the group of X.-C. Yin. The referee and the editor suggested we cite the important work of Matsu'ura (1986). Discussion with J.-C. Zhuang further clarified the significance of the simplified approach. Z.L.W. thanks the China Earthquake Administration (CEA) for assignment as the co-group-leader of the Working Group on Wenchuan Aftershocks in 2008, during the earthquake emergency action. This work is supported by the Wenchuan Fault Scientific Drilling (WFSD) project, sponsored by the Ministry of Science and Technology (MOST) of China.

Received for publication: 16 December 2011.

Revision received: 10 February 2012.

Accepted for publication: 10 February 2012.

This work is licensed under a Creative Commons Attribution NonCommercial 3.0 License (CC BYNC 3.0).

(C) Copyright C.Jiang and Z. Wu, 2012

Licensee PAGEPress, Italy

Research in Geophysics 2012; 2:e5

doi:10.4081/rg.2012.e5

introduce any additional theoretical assumptions. In practice, however, our simple approach is potentially convenient for the predictive modelers; deforming the time axis by taking the transformed time, the parameter space becomes flat or linearized.

\section{Analysis of the Wenchuan after- shock sequence}

The Wenchuan earthquake on May 12, 2008, provides an ideal example to allow the properties of the aftershock sequence to be shown. Background information about the mainshock as well as the aftershocks have been reported by Zhao and Wu. ${ }^{16}$ Aftershocks are distributed along the rupture zone, divided into two segments, namely the NE Longmenshan fault zone and the middle Longmenshan fault zone. Examining the clustering properties, i.e. properties of aftershock sequences in the surrounding region of the earthquake source, Jiang and Zhuang ${ }^{17}$ estimated the background and clustered seismicity in Yunnan and Sichuan, southwestern China, using the space-time epidemic type aftershock sequence model. Jiang and $\mathrm{Wu}^{18,19}$ discussed the effect of 
clustering properties on the predictive models of seismicity, including the accelerating moment release model and the pattern informatics algorithm.

The Wenchuan aftershock sequence shows a typical Omori-Utsu law type attenuation (Figure 1). However, convergence of the ETAS parameters is not ideal, implying that this aftershock sequence could be divided into several different stages, probably marked by the strong aftershocks. Figure 1 plots the M-T diagram of the aftershock sequence up to some 900 days after the mainshock. The earthquake catalogue is from the Monthly Earthquake Catalogue provided by the China Earthquake Networks Center (CENC), with completeness down to $M_{\mathrm{L}} 3.0 .{ }^{16}$ In the figure, the cumulative number of earthquakes (green curve) indicates a clear Omori-Utsu-law-like attenuation.

Figures 2 and 3 show the Ogata transformation for the Wenchuan aftershock sequence. As in Figure 1, this shows the events above $M_{\mathrm{L}} 3.5$ (calculated using the SASeis2006 software package). The transformed time, obtained by fitting the Omori-Utsu law (Figure 2) and the ETAS model (Figure 3), respectively, transforms from natural time axis to a distortion of the natural time axis, so that the distribution of aftershocks along this transformed time axis becomes homogeneously random. Due to the convergence feature of the ETAS model parameters, Figures 2 (A and B) and 3 (A and B) show different lengths of data to be fitted. Apparently the Omori-Utsu law fit has a better convergence, which implies that the ETAS model may have a better resolution to identify different stages of the aftershock sequence. Figures $2 \mathrm{C}$ and $3 \mathrm{C}$ provide, respectively, the analysis of the aftershock sequence before July 24, 2008, Qingchuan-Ningqiang $M_{\mathrm{S}} 6.0$ aftershock and some 2.5 months after the mainshock. The Kolmogorov-Smirnov Poisson test for the Omori-Utsu law fit (Figure 2C) and the ETAS model fit (Figure 3C) using the frequency of aftershocks (per 15 transformed time days) shows a Poisson distribution. The test can then be conducted quite simply using well known tools, such as the Molchan error diagram, ${ }^{15,20}$ along the transformed time axis with a flat parameter space.

Although the convergence behavior differs for the Omori-Utsu law fit and the ETAS fit, similarity between the transformations (Figures 2 and 3 ) indicates that it may not be necessary to use the sophisticated ETAS model for all the cases of forecast test: simple OmoriUtsu-law-based transformation may work just as well.

\section{Example application of the test of aftershock forecast: \\ the load-unload response ratio}

After the Wenchuan earthquake, enormous efforts were made both in China and abroad to try to estimate the tendency of strong aftershocks to facilitate rescue and reconstruction. After the earthquake, the public also paid special attention to the potential aftershock tendency. Approaches to the estimation of aftershock tendency range from the calculation of Coulomb failure stress change to forecast the regions with higher aftershock activity ${ }^{21}$ to the estimation of the maximum magnitude of strong aftershocks (WD Mooney, HK Gupta, GF Panza, personal communication, 2008). In this paper, what is discussed is only a special (probably the most complicated) type of aftershock forecast, that is, the forecast of the time or range of strong aftershocks.

As an example for the application of the test scheme, we show the case of the forecast based on the LURR approach. Details of the methodology and data of the forecast are described by Yin et al. ${ }^{8}$ The calculated LURR data for the forecast test are from the group of Yin. In methodology, the algorithm calculates the ratio of the number of seismic events during the Earth-tide loading phase and the unloading phase to identify the instability dur-
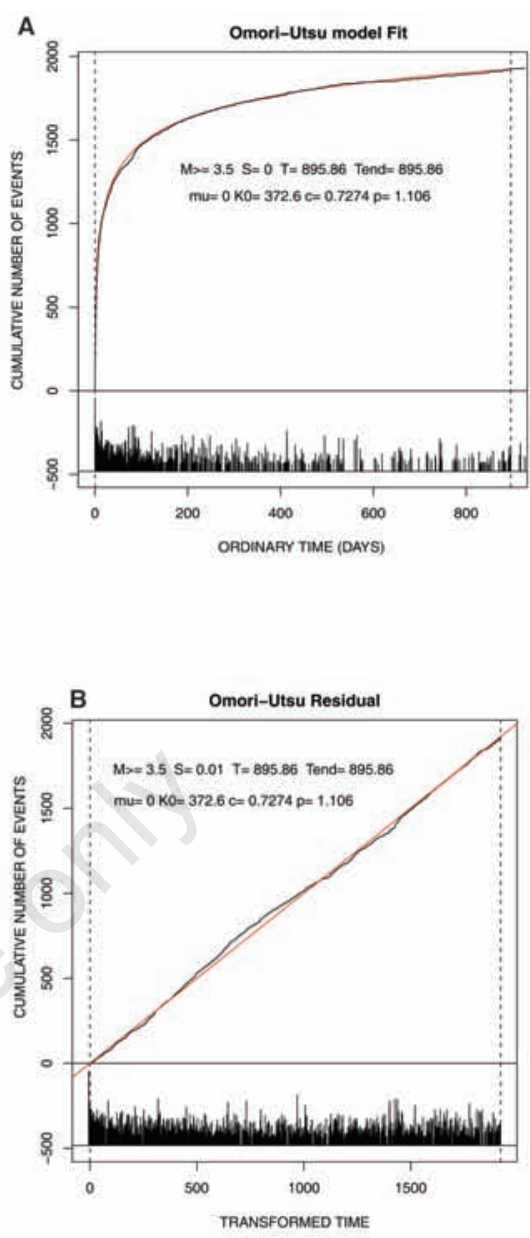

C

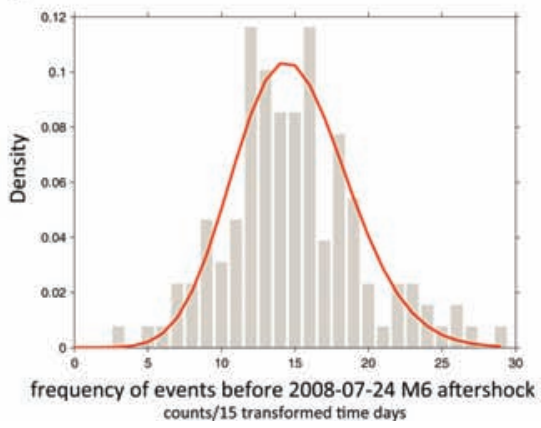

Figure 2. Transformed time analysis of the Wenchuan aftershock sequence. The Ogata transformed-time, by fitting the Omori-Utsu-law-type aftershock attenuation, transforms from natural time axis to a distortion of natural time axis, so that the distribution of aftershocks along this transformed time axis becomes homogeneously random. (A) Omori-Utsu law fit. (B) Omori-Utsu law residual. Parameters of fit are marked for each figure. (C) Omori-Utsu law fit of the Wenchuan aftershock sequence before July 24, 2008, Qingchuan-Ningqiang $M_{s} 6.0$ aftershock. Kolmogorov-Smirnov Poisson test is considered for the frequency of aftershocks (per 15 Omori-Utsu-law-transformedtime days) showing a Poisson distribution.

Figure 1. Wenchuan aftershock sequence (above $M_{\mathrm{L}} 3.5$ ) up to November 28, 2010. The green line is the cumulative number of earthquakes. 
ing the earthquake preparation. Empirically, an earthquake does not occur just at the moment when the LURR ratio dramatically increases but occurs after the LURR ratio recovers from its peak value. ${ }^{8}$ The targets for the forecasting are the TIP of the aftershocks above $M_{S} 5.0$. The forecast was conducted for

A

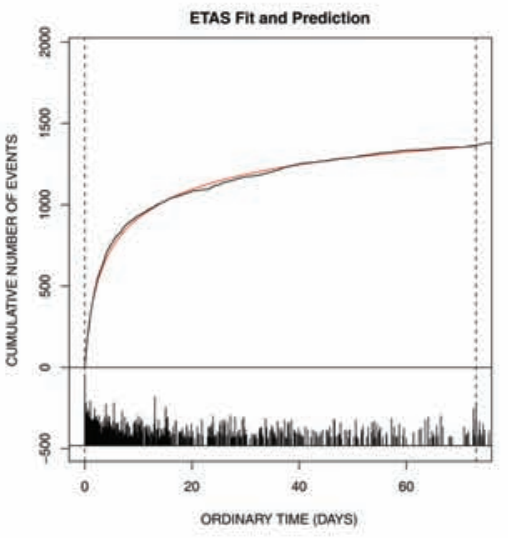

B

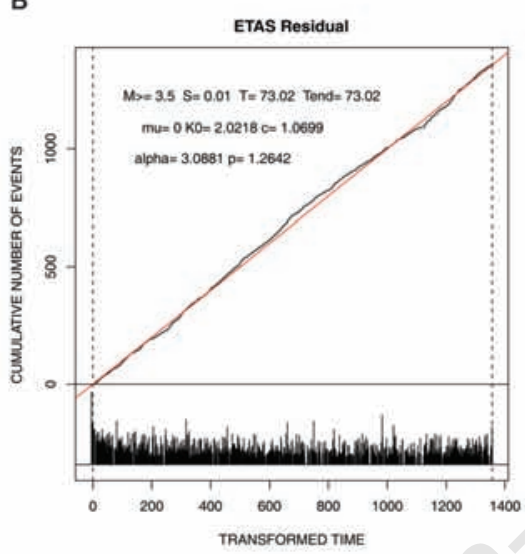

c

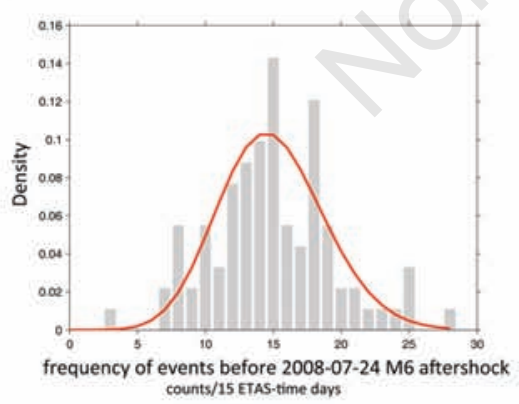

Figure 3. Transformed time analysis for the Wenchuan aftershock sequence by fitting the ETAS model. (A) ETÂS model fit. (B) Residual of the fitting. (C) KolmogorovSmirnov Poisson test for the epidemic type aftershock sequence (ETAS) of the Wenchuan aftershocks before July 24, 2008, Qingchuan-Ningqiang $M_{\mathrm{S}} 6.0$ aftershock. the two segments of the aftershock zone, namely the NE Longmenshan fault zone and the middle Longmenshan fault zone (or the SW part of the aftershock zone). As an example of the test, we consider only the forecasts for the NE Longmenshan fault zone (Figure 4).

Figure 4A and B show the normalized LURR curve and the aftershock sequence along the natural time axis, while Figure $4 \mathrm{C}$ and $\mathrm{D}$ show those along the transformed-time axis. In order to keep a constant check on the correlation between the LURR curve and the target aftershocks, we take a fixed (transformed) time delay from the time window within which the LURR ratio exceeds the anomalous thresh- old to the time window, which is expected to accommodate at least one target event. These two time windows are empirically and (somehow arbitrarily) selected as identical along the transformed-time axis. The time and time delay, which are measured along the transformed-time axis, differ slightly from the original LURR forecast. Back to natural time axis, a homogenous time delay along the transformed-time axis means that the delay time varies with time lapses: approaching backward to the origin time of the mainshock, the natural time delay is shorter and shorter. But for a long time forward, the delay time approaches homogeneous, when the trans-
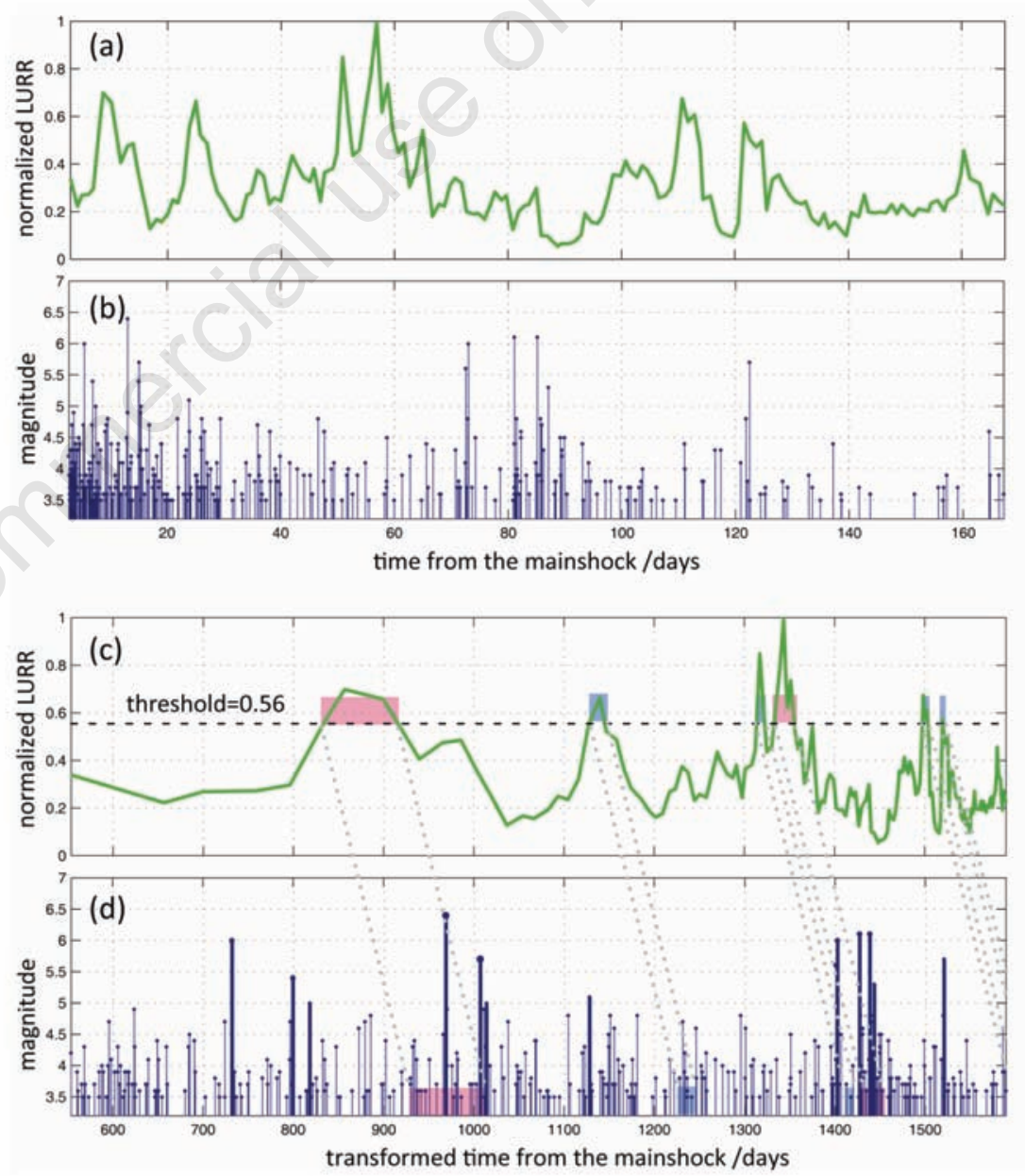

Figure 4. Load -unload response ratio (LURR)-based forecast for the Wenchuan aftershock sequence: the NE-Longmenshan fault. (A) Normalized LURR curve along the natural time axis. (B) Wenchuan aftershock sequence with magnitude no less than $M_{\mathrm{L}} 3.5$, along the natural time axis. (C) LURR curve along the transformed-time axis. (D) Aftershock sequence along the transformed-time axis. Forecasts, with delay time between the LURR anomaly and the time interval for alarm, are shown by the time ranges in pink or green, connected by the parallel dashed lines. 
spective analysis but also the real forward fore-

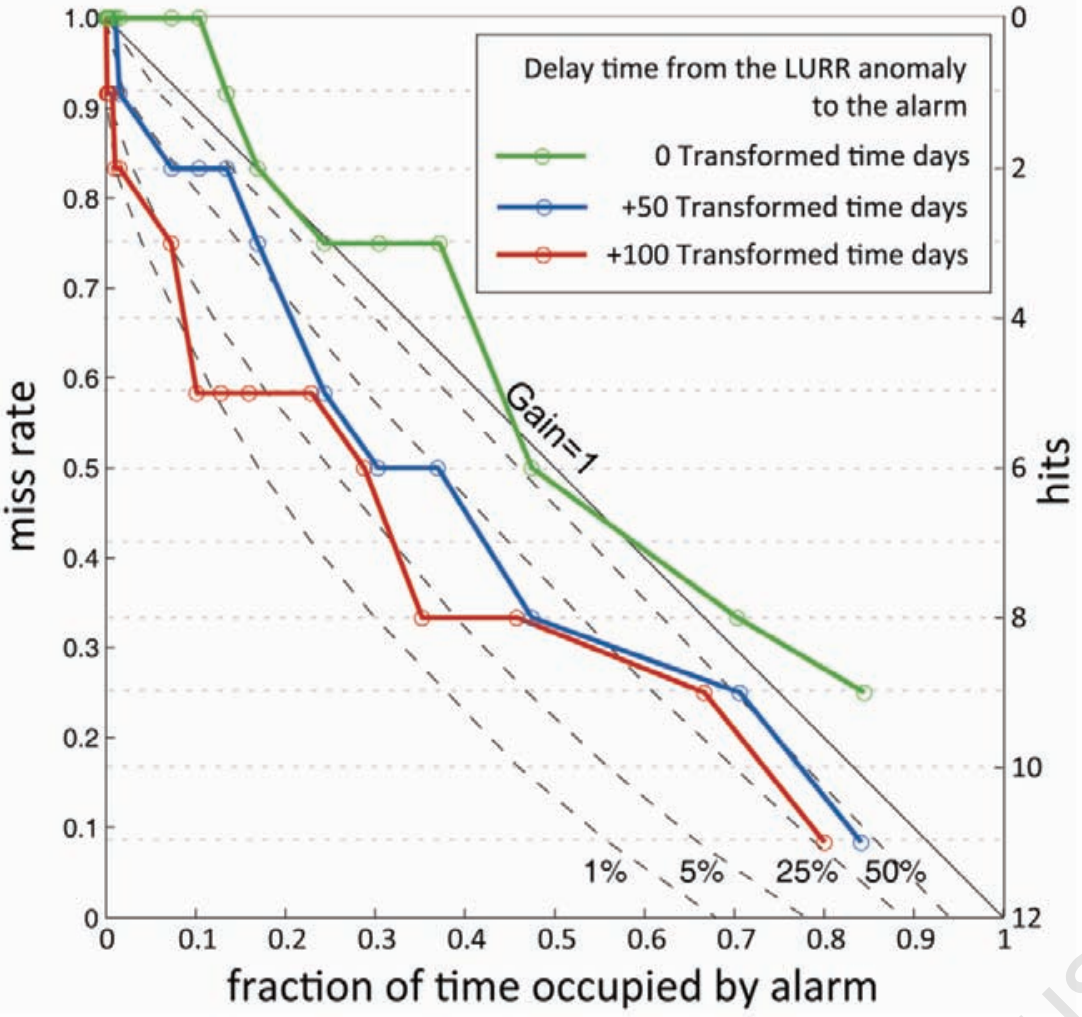

Figure 5. Molchan error diagram for the Load -unload response ratio (LURR)-based forecast, with different transformed-delay-time from the LURR anomaly to the alarm. The diagonal show the random guess with gain $=1$. Dashed lines show the theoretical curves with different gains.

formed time converges to the natural time.

Figure 5 tests the forecast with different transformed-delay times between the LURR anomaly window and the alarm time window. The anomaly threshold for the normalized LURR (0.56) is empirically taken by retrospective analysis. Due to this retrospective nature, the analysis in this paper has limited significance for the real forward forecast. ${ }^{22}$

What can be seen from Figure 5 is no more than the Molchan error diagram as usually used to test the forecast of mainshocks. The difference is that the alarm time window is measured along the transformed-time axis rather than along the natural time axis. In the figure, lines with different colors represent the forecasts with different transformed-delay times from the LURR anomaly to the alarm. As a comparison, the figure also plots the diagonal for random hit with gain equaling 1 . The result shows that the LURR-based forecasts, with delays of 50 and 100 transformed-time days, respectively, outperform random guess. The phenomena that earthquake does not occur just at the moment when the LURR ratio dramatically increases, but occurs after the LURR ratio recovers from its peak value, ${ }^{8}$ as mentioned above, is shown by the curve with zero delay time which is near to the diagonal. cast test is important for the verification of an earthquake forecast scheme, while the real forward forecast test itself is even more impor$\operatorname{tant}^{13,22}$

\section{References}

1. Jackson DD. Hypothesis testing and earthquake prediction. Proc Natl Acad Sci USA 1996;93:3772-5.

2. Kagan YY. VAN earthquake prediction - an attempt at statistical evaluation. Geophys Res Lett 1996;23:1315-8.

3. Stark PB. A few considerations for ascribing statistical significance to earthquake predictions. Geophys Res Lett 1996;23: 1399-402.

4. Stark PB. Earthquake prediction: the null hypothesis. Geophys J Int 1997:131:495-9.

5. Schorlemmer D, Gerstenberger MC, Wiemer S, et al. Earthquake likelihood model testing. Seismol Res Lett 2007;78: 17-29.

6. Michael AJ. Testing prediction methods: Earthquake clustering versus the Poisson model. Geophys Res Lett 1997;24:1891-4.

7. Utsu T, Ogata Y, Matsu'ura R. The centenary of the Omori formula for a decay law of aftershock activity. J Phys Earth 1995;43:1-33.

8. Yin XC, Zhang LP, Zhang YX, et al. The peak point of LURR and its significance. Concurrency Computat: Pract Exper 2010; 22:1549-58.

9. Rundle JB, Klein W, Turcotte DL, Malamud BD. Precursory seismic activation and critical-point phenomena. Pure Appl Geophys 2000;157:2165-82.

10. Zechar JD, Jordan TH. Testing alarmbased earthquake predictions. Geophys J Int 2008;172:715-24.

11. Ogata Y. Space-time model for regional seismicity and detection of crustal stress changes. J Geophys Res 2004;109:B03308.

12. Woessner J, Wiemer S. Assessing the quality of earthquake catalogs: Estimating the magnitude of completeness and its uncertainties. Bull Seismol Soc Amer 2005;95: 684-98.

13. Matsu'ura RS. Precursory quiescence and recovery of aftershock activities before some large aftershocks. Bull Earthq Res Inst Univ Tokyo 1986;61:1-65.

14. Ogata Y. Statistical Analysis of Seismicity updated version (SASeis2006). Computer Science Monographs (CSM), The Institute of Statistical Mathematics, Tokyo, Japan, 2006:33.

15. Molchan GM. Space-time earthquake prediction: the error diagrams. Pure Appl Geophys 2010;167:907-17. 
16. Zhao YZ, Wu ZL. Mapping the b-values along the Longmenshan fault zone before and after the 12 May 2008, Wenchuan, China, MS8.0 earthquake. Nat Hazards Earth Syst Sci 2008;8:1375-85.

17. Jiang CS, Zhuang JC. Evaluation of background seismicity and potential source zones of strong earthquakes in the Sichuan-Yunan region by using the spacetime ETAS model. Chinese J Geophys 2010;53:305-17.

18. Jiang $\mathrm{CS}, \mathrm{Wu} \mathrm{ZL}$. PI forecast with or without de-clustering: an experiment for the
Sichuan-Yunnan region. Nat Hazards Earth Syst Sci 2011;11:697-706.

19. Jiang CS, Wu ZL. Intermediate-term medium-range precursory accelerating seismicity prior to the 12 May 2008, Wenchuan earthquake. Pure Appl Geophys 2011 [Epub ahead of print].

20. Molchan GM. Earthquake prediction as a decision making problem. Pure Appl Geophys 1997;149:233-47.

21. Toda S, Lin J, Meghraoui M, Stein RS. 12 May 2008 M=7.9 Wenchuan, China, earthquake calculated to increase failure stress and seismicity rate on three major fault systems. Geophys Res Lett 2008;35: L17305.

22. Mulargia F. Retrospective validation of the time association of precursors. Geophys $\mathrm{J}$ Int 1997;131:500-04

23. Chaudhury HM, Srivastava HN. The time of occurrence and the magnitude of the largest aftershock over India. Pure Appl Geophys 1973;105:770-80.

24. Reasenberg PA, Jones LM. Earthquake hazard after a main shock in California, Science 1989;243:1173-6. 